\title{
P I6-4 I. Evidence for in vivo immune selection pressure exerted by HLA class I restricted CTL responses to anti-sense encoded HIV
} sequences

\author{
CT Berger*1, J Carlson², CJ Brumme1, ZL Brumme1, K Hartman ${ }^{1}$, LM Henry ${ }^{1}$, \\ C Kadie ${ }^{2}$, MA Brockman ${ }^{1}$, R Harrigan ${ }^{3}$, D Heckerman ${ }^{2}$ and C Brander ${ }^{4}$
}

Address: ${ }^{1}$ Infectious Disease, Ragon Institute of MGH, MIT and Harvard, Boston, MA, USA, ${ }^{2}$ Microsoft Research and Dept of Computer Science, Univ. of Washington, Seattle, WA, USA, ${ }^{3}$ BC Center for Excellence in HIV/AIDS and Div. of AIDS, Univ. of BC, Vancouver, Canada and ${ }^{4}$ ICREA and Irsicaixa-HIVACAT AIDS Res Inst, Hosp. Germans Trias I Pujol, Barcelona, Spain

* Corresponding author

from AIDS Vaccine 2009

Paris, France. 19-22 October 2009

Published: 22 October 2009

Retrovirology 2009, 6(Suppl 3):P270 doi:10.1 186/1742-4690-6-S3-P270

This abstract is available from: http://www.retrovirology.com/content/6/S3/P270

(C) 2009 Berger et al; licensee BioMed Central Ltd.

\section{Background}

Past studies have suggested that certain portions of the integrated HIV-1 proviral genome can be transcribed in the anti-sense direction, resulting in novel viral protein products that may provide alternative targets for the host anti-viral immune response. Little is known whether immune responses exist against antisense HIV peptides, whether they can recognize HIV infected cells and whether CTL-driven escape mutations within antisense peptides may be detectable on a population level.

\section{Methods}

Anti-sense sequences with potential start codons (Methionine) that preceded well-conserved genome sequences of at least 50 nucleotide triplets without stop codon were identified in 261 aligned HIV whole-genome sequences. Plasma HIV gag, pol and nef sequences from $>500$ treatment-naive, chronic infected patients were analyzed for HLA class I allele-specific viral polymorphisms in all three possible reading frames in the anti-sense direction. A maximal false-discovery rate of $20 \%$ (q value $<0.2$ ) was applied to correct for multiple tests. Polymorphisms in the anti-sense direction that affected the original coding sequence were excluded from study.

\section{Results}

Five potential anti-sense encoded proteins, including the previously described HIV anti-sense protein (ASP) were identified. Each putative protein sequence was synthesized as overlapping peptide set and was targeted by at least 2 individuals (range 2-22) of 40 HIV infected subjects tested. HLA-footprint analyses revealed 67 HLA-associated imprints (37 gag, 6 nef, $34 \mathrm{pol}$ ). For each imprint, the predicted epitopes were tested in individuals expressing the appropriate HLA allele. Several frequently-targeted antisense epitopes were identified.

\section{Conclusion}

CTL responses targeting anti-sense derived epitopes in HIV may be relatively common and may have an impact on viral evolution at the population level. Thus, T cell specificities to anti-sense encoded epitopes may be a biologically relevant mechanism contributing to immune control of HIV and may represent interesting vaccine immunogen candidates. 